\title{
Reacciones adversas a medicamentos en pacientes que acudieron a un hospital general: un meta-análisis de resultados
}

\author{
E. PUCHE CAÑAS, J. D. LUNA DEL CASTILLO'
}

\author{
Departamento de Farmacología. Unidad de Farmacología Clínica. Hospital \\ Universitario San Cecilio. ${ }^{I}$ Departamento de Bioestadística. Facultad de Medicina. \\ Universidad de Granada. Granada
}

\author{
ADVERSE DRUG REACTIONS IN PATIENTS VISITING A GENERAL \\ HOSPITAL: A META-ANALYSIS OF RESULTS
}

\begin{abstract}
RESUMEN
Objetivos: El propósito del estudio es presentar los resultados de un meta-análisis sobre las reacciones adversas a medicamentos (RAM) en pacientes ingresados y que acudieron al servicio de urgencia de un hospital general en los últimos veinte años.

Pacientes y métodos: Se hizo una revisión exhaustiva de artículos publicados entre 1985-06, ambos inclusive, en el IME (Indice Médico Español) y MEDLINE eligiendo de forma rigurosa doce de treinta publicaciones, tras aplicar criterios de inclusión y exclusión. Se requirió información sobre sintomatologia, pronóstico, tipo, fármacos implicados, imputabilidad, e ingresos por RA; los factores asociados a su aparición como la edad, sexo, número de fármacos, la historia previa de RAM, método empleado para detectar las RAM, y duración del estudio. Como método estadístico para el meta-análisis usamos el de efectos aleatorios de DerSimonian y Laird, y como programa el STATA 9.1.

Resultados: La estimación conjunta de RAM en pacientes españoles fue del 13\% (IC95\%); graves el 12\% (IC95\%); mortales el $0,1 \%$ (IC95\%); fueron ciertas el 36\% (IC95\%); probables el 49,6\% (IC95\%); del tipo A el 71\% (IC95\%). Se hospitalizaron procedentes de urgencias por RAM el 5,1\% (IC95\%). No pudimos establecer una asociación con la comorbilidad, el $\mathrm{n}^{\circ}$ de prescripciones y con la historia previa, pero si con la edad avanzada y el sexo femenino. Los sistemas más afectados por RAM fueron digestivo, piel, sistema nervioso, y cardiovascular. Entre los fármacos más implicados AINE, digoxina, IECA, antagonistas del calcio, furosemida, metamizol i.v, antidiabéticos, benzodiacepinas, broncodilatadores adrenérgicos, y antisicóticos clásicos.

Conclusiones: Las RAM son un problema médico y económico importante con aspectos por definir. Se requieren mayores esfuerzos tanto para unificar criterios de publicación de resultados en estudios observacionales sobre RAM, como precisar el papel desempeñado por algunos factores asociados a su aparición caso del sexo, la historia previa y la comorbilidad.
\end{abstract}

PALABRAS CLAVE: Reacciones adversas a medicamentos. Hospital. Urgencias hospitalarias. Pacientes hospitalizados. Metaanálisis.

\section{ABSTRACT}

Objectives: The aim of this study was to present the results of a meta-analysis on adverse drug reactions $(A D R)$ in spanish patients admitted to hospital and presenting to emergency department over the past 20 years.

Patients and methods: An exhaustive review was undertaken of relevant articles in the IME (Spanish Medical Index) and MEDLINE databases and published between 1985 and 2006, rigorously selecting 12 out of 30 publications after applying inclusion and exclusion criteria. Information was required on: symptomatology, prognosis, type, drugs involved, imputability and admissions for ADR; and factors associated with ADR onset, e.g., age, sex, number of drugs, previous history of $A D R$, method used for ADR detection, and length of study period. A random-effects model (DerSimonian and Laird) and the STATA 9.1 programme were used for the meta-analysis.

Results: The combined estimation of the percentage of Spanish patients with ADR was $13 \%$ (95\% CI); with severe ADR, $12 \%$ (95\% CI) and with fatal ADR, $0.1 \%(95 \%$ CI). ADR diagnosis was definite in $36 \%$ (95\% CI), probable in $49.6 \%$ (95\% CI), and type A in $71 \%(95 \%$ CI) of cases. Among patients in the Emergency Department for ADR, $5.1 \%$ (95\% IC) were hospitalized. No association was found with number of prescriptions or previous history. Association was established with advanced age and female sex. Digestive, skin, nervous and cardiovascular systems were those most affected by ADR. The drugs most frequently implicated included NSAIDs, digoxin, IACE, calcium antagonists, furosemide, i.v. metamizol, antidiabetics, benzodiazepines, adrenergic bronchodilators and classic antipsychotics.

Conclusions: ADRs constitute a major medical and economic problem with aspects that have yet to be defined. Greater efforts are warranted to unify criteria for the publication of results in observational studies on ARD and to determine the role played by some factors associated with their onset, e.g., sex, previous history and comorbidities.

KEY WORDS: Adverse drug reactions. Hospital. Hospital emergencies. Hospitalized patients.

Puche Cañas E, Luna del Castillo JD. Reacciones adversas a medicamentos en pacientes que acudieron a un hospital general: un metaanálisis de resultados. An Med Interna (Madrid) 2007; 24: 574-578.

\section{INTRODUCCIÓN}

El meta-análisis es una técnica estadística de revisión de datos que integra de forma cuantitativa los resultados y reduce el sesgo de selección de artículos por los autores. En la últimas dos décadas, el meta-análisis, está siendo aplicado a la revisión de estudios epidemiológicos observacionales con resultados prometedores como alternativa a las de corte narrativo, limitadas por el sesgo del autor o autores y el tamaño de muestra, a veces insuficiente para una estimación objetiva de los resultados.

Trabajo aceptado: 30 de agosto de 2007 
Las reacciones adversas a medicamentos (RAM) son un problema clínico importante que suscita la atención de médicos y autoridades sanitarias, y que frecuentemente pasa inadvertido en la clínica pudiendo evitarse al menos en la mitad de los casos. Datos procedentes de varios metaanálisis europeos y de EE.UU. indican una prevalencia de RAM en pacientes hospitalizados entre el 7-15\%; para los ingresos por esta misma causa del 3-5\%; y una mortalidad por medicamentos entre $0.05-3,7 \%(1-5)$.

En España, a pesar de la amplia bibliografía existente sobre las RAM son escasos los estudios de revisión. Nuestro grupo ha publicado recientemente uno de corte tradicional que incluyó una muestra amplia de pacientes hospitalizados y ambulatorios, hallando un porcentaje global de RAM del 3\%, siendo mayor la yatrogenia entre los pacientes que visitaron un hospital general (6). Respecto de los pacientes que acudieron a la urgencia hospitalaria por un problema relacionado con la toma de medicamentos, los datos existentes en la bibliografía española consultada dan una incidencia entre el 1,7-3,9\%, ingresando por esta causa el 6,5\% (6-8).

Dado el interés del tema nos planteamos realizar un metaanálisis de los resultados procedentes de estudios observacionales sobre las RAM en el grupo de pacientes que acudieron a un hospital, donde mayoritariamente se detectaron, al objeto de ponderar los resultados y como aportación a futuras investigaciones.

\section{TABLA I}

LISTADO DE LAS PUBLICACIONES SELECCIONADAS PARA EL META-ANÁLISIS

Rev Clin Esp 1986; 179:73-76

Med Clin (Barc) 1989; 92:530-535

An Esp Pediatr 1989; 31:49-53

Farm Clin 1992: 9:672-677

Br J Clin Pharmacol 1996; 42:319-324

Farm Clin 1996;13:586-595

Med Clin (Barc) 1998: 111:92-98 *

Br J Clin Pharmacol 1999; 47:681-688

Atención Primaria 2000; 26:42-44

Med Clin (Barc) 2003: 120:613-618

Crit Care 2003;31:694-698 *: fue tomado como dos artículos independientes por incluir adultos y
niños por separado.

\section{MATERIAL Y MÉTODO}

Se eligieron todos los artículos publicados desde el año 1985 hasta el 2006 que trataban de estudios epidemiológicos observacionales sobre detección de las RAM en pacientes españoles hospitalizados y de urgencias como tal servicio hospitalario, usando como repertorios el Medline y el IME (Indice Médico Español). De un total de treinta artículos, correspondientes a otros tantos estudios observacionales publicados fueron seleccionados de forma rigurosa doce: siete en pacientes hospitalizados y cinco procedentes de los servicios de urgencia. Definimos como reacción adversa todo signo o síntoma no deseado que aparece tras la administración de fármacos a dosis terapéuticas (OMS).

Los criterios de inclusión se basaron en que todo trabajo debería proceder de revistas con amplia difusión en repertorios nacionales e internacionales; que tuviesen normas de publicación; comité editorial; consultores externos; que definiesen a las RAM según la OMS; y que utilizasen un método de monitorización intensiva, en alguna de sus variantes, para la recogida de RAM. Se excluyeron los artículos que no cumplían los criterios de inclusión citados; aquellos que trataban de RAM inducidas específicamente por un fármaco o grupo terapéutico; y las publicaciones sobre RAM basadas en la comunicación voluntaria, incluidas las del Servicio Nacional de Farmacovigilancia.

El protocolo usado para la recogida de la información abarcó a la descripción del método; lugar y duración del estudio; población estudiada; tipo de RAM (A y B), pronóstico (leve, moderado, grave y mortal); causalidad (cierta, probable, posible y dudosa); etiopatogenia y diagnóstico de la RAM; fármacos implicados; y factores asociados a la aparición de RAM como edad, sexo, $\mathrm{n}^{\circ}$ de medicamentos, días de hospitalización y co-morbilidad.

Como los datos sobre la edad de los pacientes en los artículos seleccionados fueron rangos generales, establecimos tres categorías para su estudio: pediatría (0-17 años), adultos (18-65 años) y ancianos (más de 65 años).

\section{MÉTODO ESTADÍSTICO}

Realizado el correspondiente análisis descriptivo de los resultados, se llevó a cabo un meta-análisis usando el método de efectos aleatorios de DerSimonian-Laird (9) con un doble objetivo: obtener una estimación conjunta de los propios resultados y porque este método se deja afectar menos por la heterogeneidad entre artículos. En todos los casos se llevó a cabo el cálculo del intervalo de confianza para cada parámetro, objeto de estudio, con su error estándar correspondiente. Para realizar el análisis correspondiente se empleó el paquete estadístico STATA 9.1

\section{RESULTADOS}

Los artículos seleccionados abarcaron un total de 52.977 pacientes, el $79 \%$ procedían del servicio de urgencia, y el $20 \%$ a enfermos ingresados. Del total de pacientes el $82 \%$ fueron adultos, perteneciendo el $60 \%$ al género femenino.

La estimación conjunta de RAM hallada fue del $13 \%$ (10,4-15,6 IC95\%). En los pacientes hospitalizados fue del $8,9 \%(6,6-11,2 \%$ IC95\%), y para los que acudieron a un servicio de urgencia del 15,6\% (12,2-19\% IC95\%). La prevalencia de ingresos por RAM en pacientes procedentes del servicio de urgencia fue del 5,1\% (2,3-5,9 IC95\%)

Se catalogaron como ciertas un 3,6\% (2,1-5,2 IC95\%) y probables un 49,6\% (55,9-65,4 IC95\%); pertenecieron al tipo A, el 71\% (59,3-81,1 IC95\%). El porcentaje global de pacientes con una RAM grave fue del $12 \%(8,7-15$ IC95\%), falleciendo por esta causa el 0,1\% (0-0,03 IC95\%) (Tabla II). Entre las causas de muerte destacaron como más frecuentes la hemorragia digestiva masiva que desencadenó un shoc hipovolémico irreversible (AINE y salicilatos); y la fibrilación ventricular (digoxina sola o asociada a saluréticos). El porcentaje global de pacientes con una RAM debida a una interacción fármacológica fue del 7,3\% (14,3-1,4 IC95\%).

La prevalencia conjunta de RAM en cada una de las categorías de edad establecidas fue para pacientes pediátricos del 
TABLA II

\section{DATOS GENERALES DE PREVALENCIA DE RAM}

\begin{tabular}{lcc}
\hline & $\%$ & $I C 95 \%$ \\
\hline Pacientes totales con RAM & 13 & $9,6-14,3$ \\
Pacientes hospitalizados con RAM & 8,9 & $6,6-11,2$ \\
Que acuden a urgencias con RAM & 15,6 & $12,2-19$ \\
Ingresos debidos a RAM & 5,1 & $2,3-7,9$ \\
Graves & 12 & $8,7-15$ \\
Mortales & 0,1 & $0-0,3$ \\
Ciertas & 3,6 & $2-5,2$ \\
Probables & 49,6 & $34-65$ \\
Dudosas & 27,9 & $14,1-39,8$ \\
Tipo A & 71 & $59,3-81,1$ \\
interacción farmacológica & 7.3 & $14,3-1,4$ \\
\hline
\end{tabular}

6,1\% (2,7-9,4 IC95\%); para los adultos el 17,8\% (14,1-21,6 IC95\%); y para los ancianos el 23\% (10-46,2 IC95\%). En cuanto al sexo hallamos una estimación de RAM superior en mujeres frente a hombres, $14,4 v s .12,1 \%$ (IC95\%) con una odds ratio de 1,21 para el género femenino.

Los diagnósticos de reacciones adversas más frecuentes, tanto en pacientes hospitalizados como en los procedentes de urgencias, abarcaron signos y síntomas conocidos, esperados y frecuentes, relacionados con el digestivo, piel, sistema nervioso, cardiovascular, sangre, endocrino, respiratorio y locomotor, independientemente de la procedencia del enfermo. También se recogieron reacciones adversas infrecuentes y de pronóstico muy grave, de naturaleza alérgica y no alérgica (Tabla III).

Los grupos farmacológicos más implicados en la aparición de una RAM fueron por orden de importancia: antibacterianos (amoxicilina sola o asociada a clavulánico); AINES (inclu-

\section{TABLA III}

\section{ÓRGANOS Y APARATOS MÁS IMPLICADOS Y SINTOMATOLOGÍA MÁS FRECUENTE ASOCIADA A UNA RAM, CON UNA CASUALIDAD ENTRE CIERTA Y PROBABLE, Y DE PRONÓSTICO ENTRE MODERADO Y GRAVE}

\begin{tabular}{|c|c|c|c|}
\hline Órganos y aparatos & $\%$ & IC95\% & Cuadro clínico \\
\hline Digestivo & 39,3 & $30,7-48$ & $\begin{array}{l}\text { HD, colitis hemorrágica, pirosis, } \\
\text { dolor de estómago, diarrea }\end{array}$ \\
\hline Piel & 23,5 & $14,5-32,5$ & Toxicodermias, vasculitis, urticaria \\
\hline S. nervioso & 14,2 & $9,3-19,2$ & $\begin{array}{l}\text { Convulsiones, cefaleas, fiebre, } \\
\text { medicamentosa, somnolencia, } \\
\text { r. psicótica }\end{array}$ \\
\hline Cardiovascular & 8,3 & $4,2-12,3$ & $\begin{array}{l}\text { Arritmia, bloqueo A-V, crisis, hipo- } \\
\text { tensión arterial, shock anafiláctico }\end{array}$ \\
\hline Sangre & 2,2 & $0,8-3,70$ & $\begin{array}{l}\text { Agranulocitosis, trombocitopenia, } \\
\text { leucopenia, anemia }\end{array}$ \\
\hline Respiratorio & 1,8 & $0,7-2,9$ & $\begin{array}{l}\text { Broncoespasmo, edema glotis, } \\
\text { disnea, hipo, apnea }\end{array}$ \\
\hline Endocrino & 1,6 & $1,1-2,1$ & $\begin{array}{l}\text { Crisis de hipoglucemia, coma, hipo- } \\
\text { glucémico }\end{array}$ \\
\hline Locomotor & 0,1 & $0,1-0,2$ & $\begin{array}{l}\text { Edemas, dolores en huesos y arti- } \\
\text { culaciones, calambres en piernas }\end{array}$ \\
\hline Genito-urinario & 0,1 & $0,1-0,2$ & IRA, hematuria \\
\hline
\end{tabular}

HD: hemorragia digestiva; IRA: insuficiencia renal aguda. yendo aspirina); digoxina, IECA, antagonistas del calcio; furosemida; analgésicos puros (metamizol en aplicación i.v.); antidiabéticos (insulina y sulfonilureas); psicofármacos (benzodiacepinas y antisicóticos clásicos); broncodilatadores (salbutamol y teofilinas); y vacunas.

El tiempo empleado en realizar los estudios estuvo comprendido entre los tres y noventa y seis meses. Y el método de recogida de la información sobre reacciones adversas se basó en la monitorización intensiva o semi-intensiva de pacientes.

Una información colateral e interesante, independiente del meta-análisis, que obtuvimos tras la realización de este trabajo y que pensamos interesante decirla fue que un $60 \%$ de todas las RAM que se detectaron se catalogaron como previsibles y evitables por los autores.

No pudieron estimarse las frecuencias para el número de fármacos recibidos y días de hospitalización por estar ambos datos recogidos en solo tres artículos. Tampoco se pudieron realizar los cálculos pertinentes para la comorbilidad pues la información al respecto fue inexistente en todas las publicaciones revisadas.

\section{DISCUSIÓN}

Los resultados que presentamos además de novedosos son interesantes, pues marcan tendencias y generan hipótesis a futuras investigaciones sobre las reacciones adversas en pacientes españoles que acuden a un hospital por un problema importante de salud, además de indicar algunas sugerencias para futuras publicaciones como el papel de la comorbilidad y la mortalidad con la aparición de RAM.

Las estimaciones del porcentaje de reacciones adversas así como su tipología, pronóstico y causalidad halladas en pacientes hospitalizados como de los ingresos procedentes de un servicio de urgencia por una reacción adversa grave, fueron similares a las publicadas en otros meta-análisis (1,3-5). Sin embargo, el porcentaje global estimado de yatrogenia en pacientes que acudieron a un servicio de urgencia fue muy superior al publicado por otros autores de forma independiente $(7,8)$.

A destacar en nuestros resultados como la edad es un factor fuertemente asociado con las RAM pues en el grupo de ancianos se obtuvo un mayor número de reacciones adversas comparativamente con el resto. Estos resultados concuerdan con lo publicado en la bibliografía donde se les imputa a los ancianos un mayor porcentaje de yatrogenia grave y de ingresos por esta causa (10-15); en el origen de todo ello están la polimedicación, la comorbilidad y las mermas funcionales achacables a la vejez. En el otro extremo de la vida nuestros resultados son también concordantes con lo publicado en un meta-análisis europeo en pacientes pediátricos (16), donde las RAM son importantes tanto por su pronóstico como por las posibles secuelas con vistas a su posterior desarrollo.

Respecto del sexo, aunque nuestros resultados marcaron una cierta diferencia esta no fue lo suficiente, a nuestro juicio, para afirmar que existiera una franca asociación, concretamente entre el género femenino y la presencia de RAM. La bibliografía muestra un porcentaje mayor de mujeres con RAM frente a los varones, sobre todo para ciertos fármacos tales como: digoxina, AINE, antisicóticos antihistamínicos, gastrocinéticos, antiarrítmicos y analgésicos por causas aún no bien conocidas (17-20). En este sentido nuestros resultados confirman los de una amplia revisión sobre el tema en la que 
se concluye que el género femenino es un factor controvertido con la presencia de RAM aún no resuelto (21).

En cuanto a la mortalidad por fármacos, datos recientes indican un aumento en la última década (22), situándose para algunos autores entre la cuarta y sexta causa de mortalidad global en paises industrializados (2). Las tasas de mortalidad por fármacos procedentes de dos metaanálisis dan una prevalencia entre $0,32-5 \%$ (IC95\%) (1,2). La mortalidad global en nuestro estudio fue del uno por mil casos de RAM, y es posible que esté subestimada debido a que se registraron pocos fallecimientos por esta causa en los artículos revisados. Los datos existentes para la mortalidad debida a fármacos son aún imprecisos por lo que se requieren estudios más amplios y específicos sobre el tema dada su trascendencia en clínica.

Con respecto a la mortalidad por fármacos conviene recordar que la comorbilidad es un factor de riesgo importante cuya asociación con las RAM no ha sido suficientemente estudiada, y menos aún entre pacientes ancianos donde la polipatología y la polimedicación son habituales.

A pesar de que no pudimos establecer una relación entre el número de medicamentos recibidos y la aparición de RAM, la información procedente de ensayos epidemiológicos que la estudiaron establece una clara asociación entre ambos (6). La razón de no aportar dato alguno en este meta-análisis, estuvo en que todos los trabajos seleccionados para el estudio no reflejaron de forma ponderada dicha asociación.

En cuanto a la etiopatogenia, la mayoría de las RAM recogidas dependieron del propio efecto farmacológico (tipo A), caso de: AINE, salicilatos, anticoagulantes orales y digoxina. Una proporción baja estuvo relacionada con cuadros inesperados, raros e infrecuentes (tipo B) caso de la alergia (anafilaxia, reacción post-vacunal, toxicodermias, trombocitopenia y vasculitis); la citotoxicidad (hepatitis, nefritis y aplasia medular); y con las interacciones far- macológicas; todas ellas con un pronóstico entre grave y mortal.

Un aspecto que añade interés al conocimiento de las reacciones adversas en pacientes que usan los servicios de un hospital es el coste económico que genera la propia yatrogenia medicamentosa. Diferentes autores europeos estiman un coste promedio de $2.500 € \mathrm{x}$ paciente con RAM, incluyendo la prolongación de la estancia hospitalaria por esta causa estimada entre tres y cinco días (23-25). El coste por RAM alcanza su verdadera dimensión cuando se extrapola al total de pacientes hospitalizados que las sufren anualmente, y como ejemplo tenemos el coste de ciento treinta billones de dólares que ocasiona dicho problema en EE. UU. (26). En ninguno de los artículos revisados para este meta-análisis se halló un estudio farmacoeconómico de las RAM.

Por último, una cuestión importante e inherente a todo método de recogida de RAM es su infradetección (27). Se sabe que cuando se aplica una monitorización intensiva, en cualquiera de sus variantes, se obtiene un mayor número de RAM frente a otros métodos de detección incluida la notificación voluntaria, y esto es válido incluso para las de pronóstico grave $(28,29,30)$. Como todos los trabajos seleccionados a priori para el meta-análisis emplearon un método de monitorización intensiva, consideramos que el porcentaje de RAM no detectadas en nuestro trabajo fue bajo por causa del estricto criterio de selección de artículos.

Aunque este estudio tiene sus limitaciones consideramos que los resultados obtenidos son una aportación importante al conocimiento de las RAM en pacientes españoles que acuden a un hospital. Las reacciones adversas son un problema clínico que pasa frecuentemente inadvertido para el médico con aspectos importantes por definir, caso del papel del sexo y la mortalidad, cuyo interés sanitario, económico y social es creciente.

\section{Bibliografía}

1. Einarson TR. Drug-related hospital admissions. Ann Pharmacother 1993; 27: 832-40

2. Lazarou J, Pomeranz B, Corey P. Incidente of adverse drug reactions in hospitalizad patients. A meta-analysis of prospective studies. JAMA 1998; 279: 1200-5.

3. Beijer Hl, Blaey CJ. Hospitalizations caused by adverse drug reactions (ADR): A meta-analysis of observational studies. Pharm World Sci 2002; 24 : 46-54.

4. Wiffen P, Gill M, Edwards J, Moore A. Adverse drug reactions in hospital patients. Disponible en: www.ebandolier.com

5. Pirmohamed M, James S, Meakin S, Scott A, Walley T, et al. Adverse drug reactions as cause of admission to hospital: Prospective analysis of 18,820 patients. Br Med J 2004; 329: 15-9.

6. Puche E, Luna JD. Racciones adversas a medicamentos: Una revisión actualizada del problema en España. Rev Clin Esp 2006; 206: 336-9.

7. De Abajo FJ, Frías J, Lopo CR, Garijo B, Castro MA, Carcas A, Juárez $\mathrm{S}$, Gil A. Las reacciones adversas a medicamentos como motivo de consulta al servicio de urgencias de un hospital general. Med Clin (Barc) 1989; 92: 530-5.

8. Guemes M, Sanz E, García M. Reacciones adversas y problemas relacionados con medicamentos en un servicio de urgencia. Rev Esp Salud Pública 1999; 73: 511-8.

9. DerSimonian R, Laird N. Meta-analysis in clinical trials. Controlled Clin Trials 1986; $7:$ 177-88.
10. Walker J, Wynne H. Review: The frecuency and severity of adverse drug reactions in elderly people: Age Aging 1994; 23: 255-9.

11. Roughead E, Gilbert A, Primrose J, Sasom LL. Drug-related hospital admissions: A review of Australian studies published 1988-1996. Med J Aust 1998; 168: 405-8.

12. Manesse C, Derk FH, De Ridder M. Contribution of adverse drug reactions to hospital admission of older patients. Age Aging 2000; 29: 35-9.

13. Onder G, Pedone C, Landi F, Cesri M, Della Vedova C, Bernabei R Gambassi G. Adverse drug reactions as cause of hospital admissions: Results from the Italian Group of Pharmacoepidemiology in the Elderly (GIFA). J Am Geriatr Soc 2002; 50: 1962-8.

14. Puche E, Luna JD, García J, Góngora L. Reacciones adversas a medicamentos de pronóstico grave en ancianos institucionalizados. Rev Esp Geriatr Gerontol 2003; 38: 193-7.

15. Somers A, Petrovic M, Robays H, Bogaert M. Reporting adverse drug reactions on a geriatric ward: A pilot project. Eur J Clin Pharmacol 2003; 58: 707-14.

16. Impicciatore $\mathrm{P}$, Choonara I, Clarkson A, Provasi D, Pandolfini C, Bonati M. Incidence of adverse drug reactions in paediatric in/out patients: A systematic review and meta-analysis of prospective studies. Br J Clin Pharmacol 2001; 52: 77-83.

17. Rathore S, Wang Y, krumholz H. Sex-based differences in the effect of digoxin for the treatment of heart failure. N Engl J Med 2002; 347: 1403-11. 
18. Puche E, Aomar I, Carrillo JC. Niveles inapropiados de digoxina en sangre relativos a 2.849 pacientes procedentes de un hospital universitario: Influencia de la edad y el sexo. An Med Interna (Madrid) 2004: 21: 581-4.

19. Drici M, Clement N. Is gender a risk factor for adverse drug reactions? The example of drug-induce long QT syndrome. Dug Saf 2001; 24: 575-85.

20. Martin R, Biswas P, Freemantle S, Pearce G, Mann R. Age and sex distribution of suspected adverse drug reactions to newly marketed drugs in general practice in England: Analysis of 48 cohort studies. Br J Clin Pharmacol 1998; 46: 505-11

21. Tran C, Knowles S, Liu B, Shear N. Gender differences in adverse drug reactions. J Clin Pharmacol 1998; 38; 1003-9.

22. Eaton L. Adverse reactions to drugs increase. Br Med J 2002; $324: 8$.

23. Gautier S, Bachelet H, Bordet R, Caron J. The cost of adverse drug reactions. Expert Opin Pharmacother 2003; 4: 319-26.

24. Lundkvist J, Jönsson B. Pharmacoeconomics of adverse drug reactions. Fundam Clin Pharmacol 2004; 18: 275-80
25. Moore N, Lecointre D, Noblet C, Mabille M. Frequency and cost of serious adverse drud reactions in a department of general medicine. $\mathrm{Br} \mathrm{J}$ Clin Pharmacol 1998; 45: 301-8.

26. Jhonson JA, Bootman JL. Drug-related morbility and mortality. A cost of illness model. Arch Intern Med 1995; 155: 1949-56.

27. Belton KJ. Attitude survey of adverse drug reactions reporting by health care professionals across the European Union. The European Pharmacovigilance Research Group. Eur J Clin Pharmacol 1997; 52: 423-7.

28. Laporte JR, Arnau JM. La detección de reacciones adversas por vigilancia intensiva en pacientes hospitalizados. En: Principios de epidemiología del medicamento. Laporte JR, Tognoni G, editores. Barcelona: Salvat Editores S.A.; 1983. p. 223-5.

29. Moride Y, Aramburu F, Requejo A, Bégaud B. Under reporting of adverse drug reactions in general practice. Br J Clin Pharmacol 1997; 43: $177-81$.

30. Bäckström M, Mjörndal T, Dahlqvist R. Under reporting of serious adverse drug reactions in Sweden. Pharmacoepidemiol Drug Saf 2004; 13: 483-7. 